\title{
Suggestions for Formulating Collaborative Remote Sensing Emergency Plan Based on Case Studies
}

\author{
Binbin Liu ${ }^{\text {a }}$, Fei Wang ${ }^{\mathrm{a}, *}$, Xiaocui Zheng ${ }^{\text {a }}$, Mingyao Qi ${ }^{\text {a }}$ \\ ${ }^{a}$ Graduate School at Shenzhen, Tsinghua University, P.R.China \\ (liubb16@mails.tsinghua.edu.cn, \{wang.fei, zheng.xiaocui, qimy\}@ sz.tsinghua.edu.cn)
}

Commission III, WG III/8

KEY WORDS: Collaborative Remote Sensing Technology, Emergency Plan, Remote Sensing Services for Emergency Monitoring, Emergency Management, Disaster Management

\begin{abstract}
:
With the rapid development of the Remote Sensing (RS) technology, Remote Sensing Services for Emergency Monitoring (RSSEM) are playing a more and more important role in the field of emergency management, where the collaborative RS approaches (including such as Space-Air-Ground platforms) can provide the decision-makers a quick access to the detailed, real-time information about the emergencies. However, there are still some problems in the current mechanism of RSSEM, for example, the inappropriate choices of the collaborative RS approaches, the miscellaneous procedures and so on. It is urgent to formulate a collaborative RS emergency plan for regulating the applications of the RS monitoring approaches in order to be well prepared for the emergency management.

In our studies, creating a good collaborative RS emergency plan is the main research objective. This paper is divided into four parts. The Part I gives a brief introduction about the research background. The Part II investigates four case studies to analyze the applications of the RS technologies under the guidance of the available RS related emergency plans, and then points out the existing problems in the mechanism of the RSSEM. The Part III proposes our suggestions for formulating the collaborative RS emergency plan to explore the countermeasures of the problems pointed out in the Part II. The last part concludes this paper and discusses the future work of the collaborative RS emergency plan.
\end{abstract}

\section{INTRODUCTION}

In recent years, various types of emergencies have occurred in a high frequency all over the world including China, which seriously affects the social stability and arouses severe challenges to the emergency management. According to the incomplete statistics, in 2016, the total number of the mining accidents, explosions, fires, poison leaking incidents was up to 269 and these accidents killed more than 1130 people (Li et al, 2016a). With the features of fast acquisition, various sensors ability and advanced Space-Air-Ground platforms, the RSSEM are becoming indispensable for the emergency management, which can scientifically assist the emergency decision-making by providing the decision-makers with quick access to the detailed, real-time information about the emergencies. For instance, in the case of the 7th Aug, 2010 landslide disaster in Zhouqu, the RS technologies were applied to obtain the information about the damaged houses, broken roads and blocked rivers, timely providing rich and accurate datasets for the front line.

As is well known, the RSSEM are the important bases to support the emergency management, and the effects of which have immediate impacts on the effects of emergency response. However, most of current studies about the RS technologies stay on the technological level, but not on the point of view of the emergency plan. In March 2009, National Administration of Surveying, Mapping and Geoinformation of China (NASG) published the National Emergency Surveying and Mapping
Support Plan, and then some provinces and cities also published the plans in the provincial and municipal level. However, these provincial and municipal plans were just a little refinement of the national plan, lacking the detailed guidance of the application of the RS monitoring approaches. Therefore, the published plans are too general and unpractical for the instruction of actual emergency management operation tasks. Moreover, with the rapid development of the RS technology, there is no dynamic update in these plans, leading to the mismatch between the emergency response and the current RS approaches. This means there is still a distance between the lagging emergency plan and the actual demand for the various disasters and emergencies. In 2010 and 2016, the NASG separately published the documents on the suggestions for improving the capability of the emergency surveying and mapping support services, where it was emphasized that the collaborative RS emergency plan needed to be researched (NASG, 2016). It is crucial and urgent to study the collaborative RS emergency plan to improve the mechanism of the RSSEM in China. The reasonable collaborative RS emergency plan will improve the collaborative effect of various RS technologies and provide strong support for different stages of emergency management.

\section{ANALYSIS OF THE CASE STUDIES}

From the perspective of spatial information technology, the National Emergency Surveying and Mapping Support Plan has assisted the emergency response of China's emergencies since

* Corresponding author 
its publishment in 2009. This chapter analyzes four different types of emergencies from the point of view of the application of the available RS related emergency plans, and tries to point out the existing problems in the mechanism of the RSSEM.

\begin{tabular}{|c|c|c|c|c|}
\hline No. & Case Name & $\begin{array}{c}\text { National } \\
\text { Level }\end{array}$ & $\begin{array}{c}\text { Provincial } \\
\text { Level }\end{array}$ & $\begin{array}{c}\text { Municipal } \\
\text { Level }\end{array}$ \\
\hline & 7th Aug, 2010 & & & \\
\hline 1 & $\begin{array}{c}\text { landslide disaster in } \\
\text { Zhouqu }\end{array}$ & $\sqrt{ }$ & & \\
\hline 2 & $\begin{array}{c}\text { 29th May, } 2012 \\
\text { forest fire in } \\
\text { Changbai Mountain }\end{array}$ & $\sqrt{ }$ & $\sqrt{ }$ & \\
\hline 3 & $\begin{array}{l}\text { 18th Sept, } 2015 \\
\text { terrorist attack in } \\
\text { Baicheng }\end{array}$ & $\sqrt{ }$ & $\sqrt{ }$ & $\sqrt{ }$ \\
\hline 4 & $\begin{array}{l}\text { Penglai } 19-3 \text { oil } \\
\text { spill accident }\end{array}$ & $\sqrt{ }$ & & \\
\hline
\end{tabular}

Table 1. List of the applications of available RS related emergency plans in four cases

\subsection{7th Aug, 2010 landslide disaster in Zhouqu}

\subsubsection{Application of the available RS related emergency plans}

In 2011, Gansu Administration of Surveying, Mapping and Geoinformation published the Emergency Surveying and Mapping Plan for Serious Emergencies in Gansu Province, which covers the task of the emergency surveying and mapping support in geological disasters. However, the landslide disaster in Zhouqu happened in 2010, which means there was no collaborative RS emergency plan in Gansu Province at that time and as is shown in Table 1, the whole emergency response could only be referenced under the guidance of the National Emergency Surveying and Mapping support Plan.

\subsubsection{Application of the RS approaches}

With the help of Radarsat-2, SPOT-5, UAV and other RS approaches, the NASG quickly gained the multi-source disaster information, which was then applied to produce the 1:1000 scale high-resolution post-disaster aerial image maps. What is more, the NASG built the 3D Geographic Information System and sent disaster area topographic maps, administrative divisions and high-resolution pre-disaster maps to more than 10 departments that involved in the emergency management. The total amount of the mapping results was up to 845 pieces and total amount of the basic geographic information data was 251 GB (NASG, 2010), providing a good basis for scientific emergency management and disaster assessment.

However, in the process of the emergency response, the firsthand information acquired from the small satellites $\mathrm{HJ}-1 \mathrm{~A}, \mathrm{HJ}-$ 1B was not detailed enough to identify the landslide body. It was the satellite SPOT-5 deployed by CHARTER that played a key role in monitoring the landslide body and analyzing the cause of the disaster. In addition, due to the continuous heavy rain, optical satellites could not penetrate the clouds to monitor the ground, and the solution was to deploy Radarsat-2 satellite to gain the 3 meters resolution Radar images to make up for the needed information.

\subsection{9th May, 2012 forest fire in Changbai Mountain}

\subsubsection{Application of the available RS related emergency plans}

Table 1 also demonstrates that it was the provincial emergency surveying and mapping support plan that guided the emergency response. In 2011, the Emergency Surveying and Mapping Support Plan in Jilin Province was published. When the 29th May, 2012 forest fire in Changbai Mountain happened, the Forest Fire Prevention Headquarters of Jilin Province immediately started the Emergency Surveying and Mapping Support Plan in Jilin Province. However, this emergency plan just adds a few details on the basis of the National Emergency Surveying and Mapping support Plan, and did not perform well in terms of the integration of the emergency surveying and mapping resources in the emergency response (Qin, 2010).

\subsubsection{Application of the RS approaches}

As the main department to use the RS monitoring approaches, the Meteorological Bureau of Jilin Province applied part of the aerospace RS monitoring approaches like satellites and surveillance radars to closely monitor the weather dynamics. Furthermore, the Dunhua Air Station also sent helicopters to detect the fire spreading situation, equipped with digital cameras to take the site photos in all orientation. In this process of the emergency response, it took Dunhua Air Station totally about 8 hours to fly 5 sorties and finally Dunhua Air Station uploaded 23 fire site photos to the Forest Fire Prevention Headquarters of Jilin Province in order to monitor the fire spread situation.

From the point of view of the Air Station's rescue, the approval procedures of the aviation outfire flight was quite miscellaneous. The fire site is within $10 \mathrm{~km}$ from national boundary line, and it was after 2 hours and 40 minutes that the helicopters were approved to enter this area ( $\mathrm{Li}$ et al, 2013a). In addition, this case also exposed the shortcomings of the regular monitoring by RS technology. As the key area of forest fire prevention, the wind disaster area should have been monitored at full time and also made sure that it was the forest protection officer that discovered the first fire spot. However, in this case, the fire spot was firstly discovered by the watchman. That means when the watchman discovered the fire and smoke, the fire probably has already spread for some time. In fact, infrared RS approaches can be made full use to monitor the forest fire. Because of the large area of the forest, along with the high fire risk, it takes too much time to investigate fire hazard purely by manpower. Instead, infrared RS approaches can help monitor the real-time high risk areas, quickly find the fire spot and effectively carry out the emergency response tasks.

\subsection{8th Sept, 2015 terrorist attack in Baicheng}

\subsubsection{Application of the available RS related emergency plans}

As is shown in Table 1, in 2013, Xinjiang Bureau of Surveying, Mapping and Geoinformation published the Emergency Surveying and Mapping Support Plan in Xinjiang. According to this emergency plan, the departments of Xinjiang Bureau of Surveying, Mapping and Geoinformation formulated a more 
detailed one to assist the decision making. However, the terrorist attack happened in depopulated zone where there was little basic geographic information because of the special geography. In that case, even with the RS related emergency plans, the emergency response lasted for 56 days.

\subsubsection{Application of the RS approaches}

The terrorist attack in Baicheng happened in depopulated zone at an altitude of 3000 meters. Due to the complex terrain, poor basic geographic information and tele-communication infrastructure, there were almost no map products applied during this emergency response. Additionally, the harsh environment made it impossible to apply the UAV to perform aerial photography and reconnaissance missions. Therefore, it was the mutual help between the professionals and the local people that played the important role in search of the terrorists. Some local herdsmen plotted suspicious locations on the handpainted maps provided by the Xinjiang Forestry Administration, forming a search and siege route plan along with the SWAT soldiers.

The emergency response of this terrorist attack lasted for 56 days and it well reflected that the lack of the basic geographic information was the fatal problem. Thus, the Xinjiang Bureau of Surveying, Mapping and Geoinformation should not only prepare RSSEM well for the urgent time, but also enhance the accumulation of the basic geographic information. For example, satellites can be applied to acquire the periodic image information in depopulated zone, and then the departments can timely interpret and create specialized map products for emergent needs.

\subsection{Penglai 19-3 oil spill accident}

\subsubsection{Application of the available RS related emergency plans}

In July 1, 2011, the Shandong Provincial Institute of Land Surveying and Mapping published the Emergency Surveying and Mapping Support Plan in Shandong Province. However, the Penglai 19-3 oil spill accident happened in June 4, 2011, just several days before the publishment. As shown in Table 1, there were no available provincial RS related emergency plans to be the guidance in this case.

\subsubsection{Application of the RS approaches}

After the Penglai 19-3 oil spill accident, the State Oceanic Administration (SOA) immediately conducted the deployment of satellite, aerial photography, marine surveillance ship and other approaches to make sure the all-weather field monitoring. In general, the SOA completed interpretations of 73 scenes of the RS monitoring data acquired from the deployment of 8 ships with a total sail more than 30 thousand miles and 2 planes with a total flight more than 24 thousand kilometers.

Environmental monitoring in Bohai has always been the top priority of China's marine environmental protection work, but the SOA did not notice the oil leakage in time until the ConocoPhillips itself reported the urgent situation. To some extent this phenomenon reflected the insufficient application of the RS monitoring approaches in the prevention and preparedness stage.

\subsection{Summary}

Through the four case studies described above, it can be found that a variety of RS technologies play an irreplaceable role in helping decision makers to master the field information in every stage of the emergency management. But at the same time, these cases also reflected some loopholes in the current mechanism of the RSSEM.

Firstly, the current mechanism of the RSSEM had the problem about inappropriate choices of the RS technologies. In 7th Aug, 2010 landslide disaster in Zhouqu, the inappropriate choice of the satellites resulted in the delay of the next emergency response. In the 29th May, 2012 forest fire in Changbai Mountain, the fire was not discovered in time because of lacking the infrared RS monitoring approaches. In the 18th Sept, 2015 terrorist attack in Baicheng, the pre-selected RS monitoring approaches were limited by geographical conditions. In the Penglai 19-3 oil spill accident, the key areas lacked suitable RS monitoring approaches to be monitored in a medium term. In fact, such phenomena are quite common in many other cases in the stage of the emergency management. And to a certain extent, not using the available RS monitoring approaches or using inappropriate ones may both result in the waste of the time and the cost.

Secondly, the current mechanism of the RSSEM had the problem about the ill-defined or miscellaneous procedures. In the 29th May, 2012 forest fire in Changbai Mountain, the helicopters kept hovering in the air for several hours because of the approval procedures. Actually, not only the helicopters, but also the ground survey vehicles like cars are limited to be used by the implicit procedures defined in the emergency plan. Therefore, how to ensure the efficient deployment of RS monitoring approaches is a major problem that needs to be solved urgently.

\section{SUGGESTIONS FOR FORMULATING COLLABORATIVE RS EMERGENCY PLAN}

According to the problems reflected by the four case studies and from the point of view of the quick match and the efficient deployment about the optimum RS monitoring approaches, this chapter proposes suggestions for formulating collaborative RS emergency plan to guarantee the efficiency of the emergency management.

\subsection{Establish the Quick Match Mechanism of Optimum RS Technologies}

Firstly, the efficient collaborative RS emergency plan needs to establish the quick match mechanism of optimum RS technologies, which can well solve the problem about the inappropriate choices of the RS technologies. When the emergencies happen, the main solution is to comply with the experts' experience or the domain knowledge. But these results of the selection are drastically subjective and may not be optimum. Thus many researchers investigate it, using the various matches between the RS platforms and sensors to choose the optimum RS approaches (Toth C et al, 2016a).

As described in the four cases above, different kinds of emergencies in different stages of the emergency management may have different requirements of RS technologies. As shown in Figure 2, in the process of the emergency preparedness, the 
landslide disaster needed satellites working with geometric high resolution sensors (HRG) to obtain the images to monitor the situation of the landslide body, while the forest fire needed satellites working with infrared multispectral sensors to monitor the situation of the fire. Even in the same stage of the emergency management and deploying the same platform, different kinds of sensors are employed due to different kinds of emergencies. As represented by another example, in the process of the emergency response, the anti-terrorism needed UAV to acquire the geography data to search for terrorists, while the oil spill accident needed marine surveillance ships and satellites to monitor the oil diffusion range. It is obvious the platforms in these two emergencies are different. From this point of view, different RS platforms equipped with different sensors will produce various practical effects. Therefore, it is meaningful to carry out in-depth study for RSSEM from the perspective of the platforms and sensors.

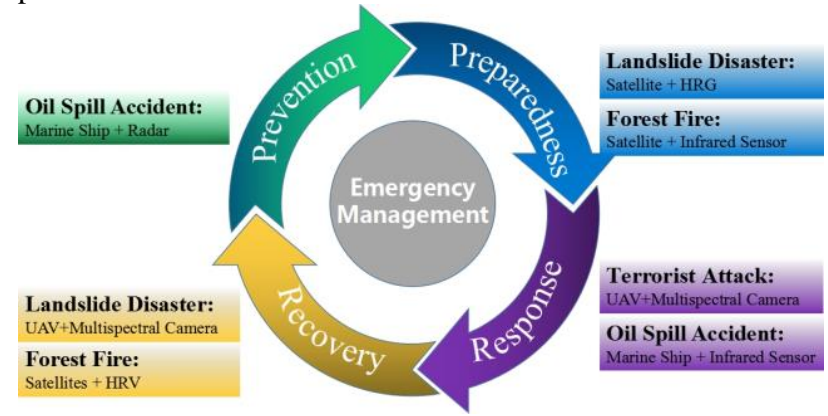

Figure 1. Applications of platforms equipped with RS sensors in different emergency management stages in the four case studies

The RS platforms usually can be divided into space RS platforms, aerial RS platforms and ground RS platforms. According to the geo-location, the sensors can be divided into space-based sensors, aerial-based sensors, ground-based sensors, underground-based sensors, underwater-based sensors and creature-based sensors (Wang et al, 2010a). The diversity of the RS platforms and the sensors makes the RS technologies play the tremendous role in the emergency management. On the basis of the matching theory of RS platforms and sensors, some researchers put forward an emergency ontology model, which works with RS monitoring resources model, to give the optimum RS technologies quickly. This match mechanism commences from the features of emergencies and also takes the RS monitoring resources into consideration. It can help the decision-maker at the very start to acquire appropriate RS data and save the valuable time for next decision or rescue.

\subsection{Establish a High-efficiency RS Technologies Scheduling Mechanism}

In regard to the emergencies, time is everything, which means establishing the match mechanism is far from enough. A highefficiency RS tasks scheduling mechanism is also needed to be included in the collaborative RS emergency plan. In the 29th May, 2012 forest fire in Changbai Mountain, it took 2 hours and 40 minutes for the helicopters to conduct the forest fire fighting and rescue mission. The miscellaneous procedures greatly weakened the high timeliness of RS monitoring approaches, being the fatal problem in the emergency response. To solve this problem, flat management mechanism is a good choice. The flat management mechanism is mainly based on decentralized management. It moves down the right center and ensures the relative independence between each primary organization, which directly reduces the delay of the decision making and improves the efficiency of emergency response (Song et al, 2003a). In 1999, the U.S.A used flat management mechanism in its RS Standard Operating Procedures (SOP). In this SOP, the government is the leading force, but it also sets up the specialized coordination departments or groups to deploy the RS technologies, which makes the scheduling procedures clear and fast. Although the current mechanism of the RSSEM in China has stipulated the leading bodies, offices, working institutions, local institutions and social forces in the emergency management, there are still some delay and distortion problems in the information transmission because of the lack of specialized coordination departments. Carrying out the flat management by adding specialized coordination person in charge of the scheduling procedures to each RS related department can well solve these problems. For example, when the emergencies happen, the specialized coordination persons are $7 * 24$ on duty to take charge of the RS coordination and the independent submission of needed geospatial information, which supports the whole emergency management.

\section{CONCLUSIONS AND PERSPECTIVES}

The well-defined collaborative RS emergency plan can play an important guiding role in the emergency management. It is of great significance to study the collaborative RS technologies as well as the emergency plan issue, in order to constantly improve the efficiency of the RSMEM in the process of emergency management. Through summarizing four emergency cases in this paper, problems of the current mechanism of the RSSEM have been pointed out, such as inappropriate choices of the RS technologies and the miscellaneous procedure. Then this paper proposes two suggestions for formulating collaborative RS emergency plan to improve both the match mechanism and the scheduling mechanism. Although our given suggestions in this paper are not comprehensive for defining a full covering emergency plan, they are very enlightened and can serve well for RS or emergency management professionals when performing emergency plan related tasks (including creating the new one, revising, practicing and etc.).

Now driven by IT technology and disaster modelling and simulation technology, the technology of emergency plan is evolving into so called digital emergency plan technology, which is regarded as the best way to improve the emergency plans' scientificalness, effectiveness and pertinence (Zhang et al, 2010a). Meanwhile, with the fast development of RS technology, the quick optimum RS technologies matching method for specific emergencies will have new prospects. With the optimization of organizational structure in geospatial related government sectors, the coordination mechanisms for the RSSEM will definitely improved. Therefore, it can be imagined in the future that with the guidance of the well-defined collaborative RS emergency plan, various RS technologies can be applied appropriately at different stages of emergency management. And most emergency management tasks can be implemented in an efficient and ordered way.

\section{ACKNOWLEDGEMENTS}

This paper is funded by National Key Research and Development Program of China (Grant No. 2016YFB0502601 and Grant No. 2016YFC0803107) and Shenzhen Technology Innovation Program (Basic Research JCYJ20170307152553273). 


\section{REFERENCES}

Li, S., Xiao, L., 2016a. Statistics of industrial accidents in China in 2016. Journal of Safety and Environment, (02-06), pp. 395-396.

Li, S., Zhang, R., 2013a. Fire fighting experience of "5.29" forest fire in Changbai Mountain National Nature Reserve. Forest Fire Prevention. (2), pp. 39-42.

NASG, 2010. Surveying and Mapping Departments to Serve Zhouqu Landslide Disaster http://chzt.sbsm.gov.cn/article/chfxcr/tenchfxcr/chfwbz/201008/ 20100800071831.shtml (13 Aug. 2010).

NASG, 2016. Suggestions of the National Administration of Surveying, Mapping and Geoinformation of China on Further Strengthening the Construction of Emergency Surveying and Mapping Services http://www.gdemo.gov.cn/zwxx/zcfg/gjzcwj/gjbmwj/201611/t2 0161114_242536.htm (19 Sep. 2016).

Qin, Q., 2010. The Study of the Problems and Countermeasures in Surveying and Mapping Emergency support in Jilin Province. Jilin University.

Song, J., Yin, H., 2003a. Flat management theory and the reform of government institutions in China. GUANGXI Social Science, (12), pp. 16-18.

Toth, C., Jóź ków, G., 2016a. Remote sensing platforms and sensors: A survey. Isprs Journal of Photogrammetry \& Remote Sensing, 115, pp. 22-36.

Wang, F., Yuan, H., 2010a. Challenges of the Sensor Web for disaster management. International Journal of Digital Earth, 3(3), pp. 260-279.

Zhang, C., Pei, Y., Qiu, H., 2010a. Current status and development trend of digital emergency plan at home and abroad. Journal of Safety Science and Technology, 06(5), pp. 154-158. 